\title{
Messung der Ionentemperatur mit Hilfe des thermischen Doppler-Effekts an einer Wasserstoff-Lichtbogenentladung
}

\author{
D. LUDWIG und J. RAEDER \\ Institut für Plasmaphysik, Garching bei München \\ (Z. Naturforsch. 25 a, 473-481 [1970]; eingegangen am 22. Dezember 1969)
}

\begin{abstract}
The radial ion temperature distribution in a 2600 amp hydrogen arc in a $30 \mathrm{kG}$ axial magnetic field was measured spectroscopically with a Fabry-Perot interferometer by evaluating the half width of Doppler broadened carbon lines. As the admixture of methane had to be low, in order to prevent a strong reduction of the temperature on the axis, the intensities of the C III and C IV lines were relatively weak. Consequently an interference filter with narrow transmission band width was used instead of a spectrograph in front of the F.-P.-interferometer.

The broadening of the spectral lines, which were used for measurement, was caused mainly by the thermal Doppler effect. The rotation of the plasma and the macroscopical Doppler effect resulting therefrom did not disturb the measurements. The ion temperature, which was found to be $1.4 \times 10^{5}{ }^{\circ} \mathrm{K}$ on the axis, was determined from the half width of the profiles of a C III spectral line.

Since the discharge was observed side-on, the measured integrated values were reduced to radial profiles by using appropriate inversion formulae.
\end{abstract}

Treten in einer Lichtbogenentladung sehr große radiale Temperaturgradienten auf, so kann die Bestimmung der Temperatur aus dem Intensitätsverhältnis von Spektrallinien aufeinanderfolgender Ionisationsstufen eines Elementes dann zu großen Fehlern führen, wenn man für das Ionisationsgleichgewicht eine "statische“ Ionisationsformel zugrunde legt. Dies haben Bergstedt ${ }^{1}$ und Tichmann ${ }^{2}$ an Lichtbogenentladungen demonstriert, denen ein starkes axiales Magnetfeld überlagert war. Durch das Magnetfeld werden die radialen Wärmeleitungsverluste so stark reduziert, daß bei relativ geringen elektrischen Leistungen Achsentemperaturen oberhalb $10^{5}{ }^{\circ} \mathrm{K}$ im stationären Betrieb erreicht werden. Da der Durchmesser dieser Entladungen nur wenige Zentimeter beträgt, bilden sich große radiale Gradienten von Temperatur und Teilchendichten aus. Treten nun hohe Relativgeschwindigkeiten zwischen den verschiedenen ionisierten Plasmakomponenten auf, so führen die endlichen Rekombinationszeiten zu falschen Werten für die Elektronentemperatur. Daher war es von großem Interesse, an einem Wasserstofflichtbogen die Temperatur mit einer anderen - von der Einstellung des lokalen Ionisationsgleichgewichtes unabhängigen - Methode zu bestimmen. Hier bot sich die Ausnutzung des thermischen Doppler-Effektes an geeigneten Spektrallinien an. Diese Methode hat außerdem den Vorteil, daß man un-

Sonderdruckanforderungen an Dipl.-Phys. D. LuDwiG, In stitut für Plasmaphysik, D-8046 Garching bei München. mittelbar die Temperatur der Ionen mißt und durch Vergleich mit Messungen der Elektronentemperatur feststellen kann, ob sich ein lokales thermodynamisches Gleichgewicht zwischen leichten und schweren Teilchen eingestellt hat.

Voraussetzung für dieses Verfahren ist die Emission von Spektrallinien, deren Verbreiterung ganz überwiegend vom thermischen Doppler-Effekt hervorgerufen wird und bei denen man andere Verbreiterungsmechanismen eindeutig vom Doppler-Effekt trennen kann.

Der Wasserstoff in dem untersuchten Lichtbogen ist bei den zu erwartenden Temperaturen vollständig ionisiert und sendet daher aus der Umgebung der Achse keine Linienstrahlung mehr aus. Er kommt deshalb für Doppler-Messungen nicht in Frage, d. h. man muß dem Wasserstoff ein Element zumischen, das bei Temperaturen von ca. $10^{5}{ }^{\circ} \mathrm{K}$ noch Linien emittiert.

\section{Beschreibung des Lichtbogens}

Die Messungen werden an einem Wasserstoff-Lichtbogen durchgeführt, der in einem axialen Magnetfeld brennt (Abb. 1). Der Wolfram-Kathode steht eine ringförmige $\mathrm{Cu}$-Anode gegenüber. Zwischen beiden Elektroden befindet sich eine ebenfalls aus Kupfer bestehende Ringdüse, die auf Anodenpotential liegt und durch die der Bogen hindurchbrennt. Die gesamte Elek-

1 K. Bergstedt, Z. Naturforsch. 24 a, 299 [1969].

2 K. Tichmann, Bericht des Inst. f. Plasmaphysik, IPP $3 / 77$. 


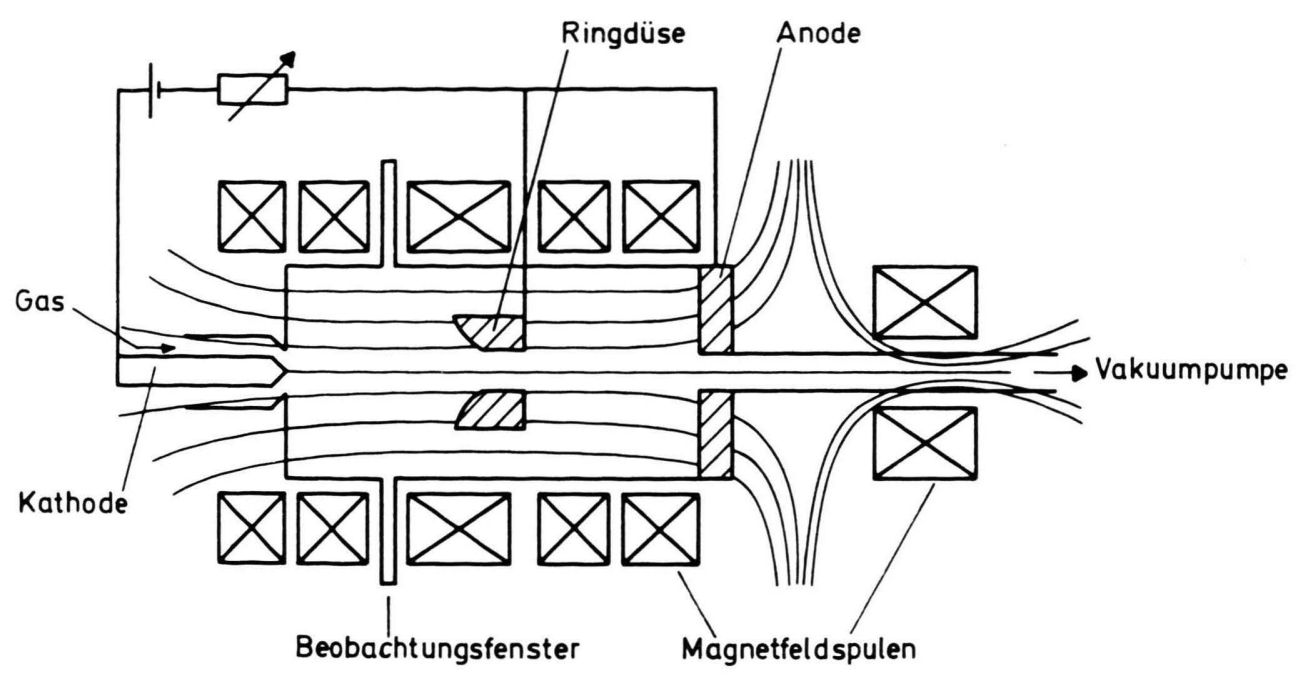

Abb. 1. Experimentelle Anordnung.

trodenanordnung ist in einem Vakuumkessel aus V2AStahl befestigt. Vier jeweils um $90^{\circ}$ versetzte Beobachtungsfenster können für die optischen Messungen ausgenutzt werden.

Die effektive Bogenlänge (Kathode - Ansatzpunkt des Bogens in der Anode) beträgt $80 \mathrm{~cm}$. Die Ringdüse ist $15 \mathrm{~cm}$ von der Kathode entfernt, während der Abstand von der Kathode zur Mitte der Beobachtungsfenster $9 \mathrm{~cm}$ beträgt.

Gezündet wird die Entladung durch Anlegen einer Gleichspannung von $1 \mathrm{kV}$ zwischen Anode und Kathode bei einem Kesseldruck von etwa 0,5 Torr. Der Stromverlauf ist so programmiert, daß nach der Zündung für die Dauer von 0,4 sec ein Bogenstrom von $600 \mathrm{~A}$ fließt, der dann für die Dauer von $0,8 \mathrm{sec}$ auf seinen Maximalwert von 2600 A hochgeschaltet wird (Abb. 2).

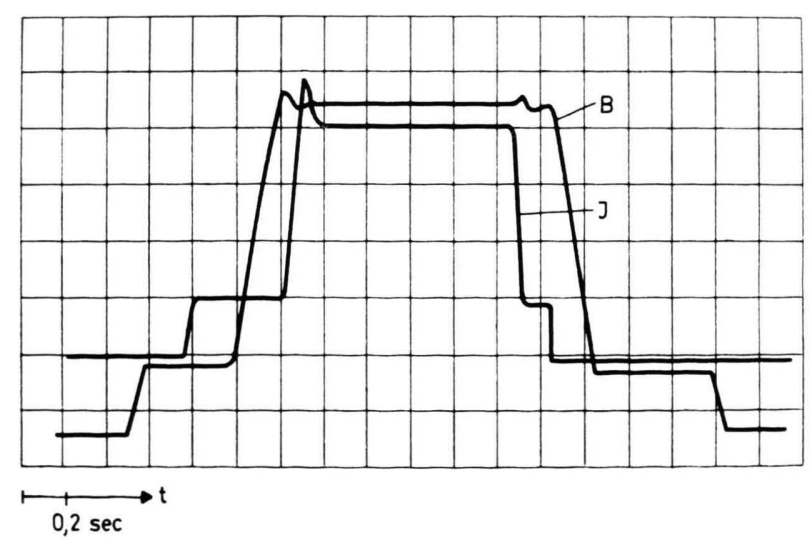

Abb. 2. Zeitlicher Verlauf von Strom $I$ und Magnetfeld $B$.

Der Verlauf der magnetischen Feldlinien ist qualitativ in Abb. 1 dargestellt. Durch ein magnetisches Gegenfeld am hinteren Ende des Kessels wird ein reproduzierbarer Stromansatz an der Anode erreicht. Ohne dieses Gegenfeld, das dem Entladungsstrom die Möglichkeit gibt, parallel zum Magnetfeld die Anode zu erreichen, endet die Entladung häufig auf dem Anodenflansch oder der Ringdüse.

Um die verschiedenen Messungen miteinander vergleichen zu können, werden folgende Betriebsdaten bei allen Untersuchungen eingehalten:

Bogenstrom: $2600 \mathrm{~A}$, Magnetische Induktion : $32 \mathrm{kG}$, Gaszusammensetzung: $\mathrm{H}_{2}+3,9 \% \mathrm{CH}_{4}$.

Bei diesen Bedingungen stellte sich stationär ein Bogen mit einem optisch sichtbaren Durchmesser von $25 \mathrm{~mm}$ ein.

\section{Auswahl geeigneter Spektrallinien}

Für die relative Intensitätsverteilung in einer Doppler-verbreiterten Spektrallinie gilt :

$$
\begin{array}{r}
\frac{\mathrm{d} I}{I}=\frac{\mathrm{J}}{\sqrt{\pi}} \exp \left\{-\left(\frac{\Delta \omega}{\Delta \omega_{\mathrm{D}}}\right)^{2}\right\} \frac{\mathrm{d} \omega}{\Delta \omega_{\mathrm{D}}} \\
\text { bzw. } \frac{\mathrm{d} I}{I}=\frac{1}{\sqrt{\pi}} \exp \left\{-\left(\frac{\Delta \lambda}{\Delta \lambda_{\mathrm{D}}}\right)^{2}\right\} \frac{\mathrm{d} \lambda}{\Delta \lambda_{\mathrm{D}}}
\end{array}
$$

$\left[\Delta \hat{\lambda}_{\mathrm{D}}=\left(\lambda_{0} / c\right) \sqrt{2 R T / \mu}, R=\right.$ allgemeine Gaskonstante, $c=$ Lichtgeschwindigkeit, $\mu=$ Atomgewicht, $\lambda_{0}=$ Wellenlänge der jeweiligen Spektrallinie $]$.

Daraus folgt für die ganze Halbwertsbreite der Linie

$$
2 \Delta^{\prime} \lambda=\frac{\lambda_{0}}{c} 2 \sqrt{\ln 2} \sqrt{2 R T / \mu}
$$

und für die Temperatur der strahlenden Teilchen

$$
T_{i}=\frac{\mu}{2 R}\left(\frac{2 c \Delta^{\prime} \lambda}{2 \sqrt{\ln 2} \lambda_{0}}\right) .
$$

Bei den erwarteten Temperaturen in der Größenordnung von $10^{5} \mathrm{~K}$ und einer Wellenlänge $\lambda_{0}=$ 
$5000 \AA$ ergibt sich nach Gl. (3) für Kohlenstoff die Halbwertsbreite $2 \Delta^{\prime} \lambda=0,33 \AA$.

Diese Zahl soll unserer weiteren Diskussion zugrunde gelegt werden. Wir werden alle maßgeblichen Verbreiterungsmechanismen, die in unserem Plasma eine Rolle spielen, gegen sie abschätzen.

Aus der Theorie der Stark-Verbreiterung durch Mikrofelder und aus vielen experimentellen Untersuchungen ergibt sich, daß die Wasserstofflinien bei Dichten von einigen $10^{15} \mathrm{~cm}^{-3}$ breiter als $1 \AA$ sind, also nicht für Doppler-Messungen in Frage kommen. Außerdem ist der neutrale Wasserstoff im Innern des Bogens bei den erwartete Temperaturen bereits „ausgestorben“, so daß er für die Bestimmung der Achsentemperatur nicht verwendet werden kann.

Um die Gastemperatur auch im Bogenzentrum messen zu können, muß man dem Wasserstoff ein Element beimischen, das bei den Temperaturen im Innern des Bogens noch Linien emittiert. Da die Doppler-Verbreiterung mit abnehmendem Atomgewicht zunimmt (proportional $1 / \sqrt{\mu}$ ), muß man leichte Elemente auswählen, die sich dem Wasserstoff einfach zumischen lassen und bei denen der Doppler-Effekt der dominierende Verbreiterungsmechanismus ist. Wegen der einfachen technischen Ausführung bietet sich dafür Kohlenstoff an, der sich in Methan $\left(\mathrm{CH}_{4}\right)$ dem Wasserstoff hinzufügen läßt. Versuche ergaben, daß im Zentrum des Lichtbogens C III- und CIV-Linien emittiert werden. Eine Abschätzung störender Einflüsse auf die Form dieser Linien führt zu folgenden Ergebnissen:

\section{a) Stark-Aufspaltung}

Die beobachteten elektrischen Felder betragen nach ${ }^{3}$ axial etwa $3 \mathrm{~V} / \mathrm{cm}$ und radial bis zu $400 \mathrm{~V}$ $/ \mathrm{cm}$. Daraus ergibt sich eine maximale Wellenlängenverschiebung von $1,6 \cdot 10^{-2} \AA$, ein Wert, der klein gegen den Doppler-Effekt ist.

\section{b) Hyperfeinstruktur}

Die vom Kernmoment durch Kopplung mit dem Bahndrehimpuls und Spin des Elektrons erzeugte Termaufspaltung ist dem Kernspin $i$ proportional. Bei $\mathrm{C}^{12}$ ist $i=0$, d. h. die Terme spalten nicht auf.

3 C. Mahn, H. Ringler u. G. Zankl, Z. Naturforsch. 23 a, 867 [1968].

4 A. Unsöld, Astronomische Mitteilung 214, Kiel 1943.

5 K. Bockinasten, Ark. Fysik 10 (Nr. 40), 568 [1956].
Ein weiterer Grund für eine Hyperfeinstruktur ist die Isotopenverschiebung der Energieterme eines Atoms oder Ions. Da jedoch bei Kohlenstoff das stabile Isotop $\mathrm{C}^{13}$ nur einen Anteil von $1,1 \%$ hat, können wir auch diesen Effekt vernachlässigen.

\section{c) Stark-Verbreiterung}

Allgemeingültige quantitative Aussagen über den Stark-Effekt bei C III- und C IV-Linien lassen sich nicht machen. Man muß die Verbreiterung der jeweiligen Spektrallinien nach quantenmechanischen bzw. klassischen Arbeiten, z. B. von UNSÖLD ${ }^{4}$, BocKKASTEN $^{5}$ und Murakawa und Yamamoto ${ }^{6}$ gesondert abschätzen. Für die Linien C III $5696 \AA$ und C IV Dublett $5801 / 5812 \AA$, die auch BergstedT ${ }^{1}$ für Doppler-Messungen verwendet hat, ergeben sich Stark-Verbreiterungen in der Größenordnung von $10^{-3} \AA$.

\section{d) Zeeman-Verbreiterung}

Zeeman-Verbreiterung tritt auf, wenn Linien aus Gebieten mit verschiedener magnetischer Feldstärke emittiert werden.

Nach Wienecke ${ }^{7}$, Raeder und WirTZ ${ }^{8}$ erhält man in Lichtbögen, denen ein achsenparalleles Magnetfeld überlagert ist, einen Druckanstieg in der Bogensäule. Da die Druckdifferenz durch eine Magnetfeldänderung aufrechterhalten wird, emittiert das Plasma aus einem Gebiet, in dem sich das Magnetfeld räumlich kontinuierlich ändert. Mit unseren experimentellen Werten: Außendruck $p_{\mathrm{a}}=2$ Torr, äußeres Magnetfeld $B_{0}=32 \mathrm{kG}$ ergibt sich bei einer Achsentemperatur von $10^{5} \mathrm{~K}$ ein Druck von etwa $2,5 \cdot 10^{5} \mathrm{dyn} / \mathrm{cm}^{2}$ (=190 Torr) und eine Magnetfeldänderung über den Bogenradius von $100 \mathrm{G}$. Daraus folgt eine Zeeman-Verbreiterung von $3 \cdot 10^{-3} \AA$. Dieser Wert liegt nur eine Größenordnung über der natürlichen Linienbreite und kann deshalb unberücksichtigt bleiben.

\section{e) Zeeman-Aufspaltung}

Durch Einfluß eines äußeren Magnetfeldes spalten die Energieniveaus der Spektrallinien auf. Die Wellenlängenverschiebung der einzelnen Zeeman-Kom-

\footnotetext{
6 K. Murakawa u. M. Yamamoto, J. Phys. Soc. Japan 20, 1057 [1965].

7 R. WienECKE, Z. Naturforsch. 18 a, 1151 [1963].

8 J. Raeder u. S. Wirtz, Z. Naturforsch. 23 a, 1695 [1698].
} 
ponenten lassen sich berechnen (z. B. ${ }^{9}$ ). In ${ }^{1}$ sind die Aufspaltungsbilder für das C IV-Dublett 5801 $/ 5812 \AA$ bei einem Magnetfeld von $50 \mathrm{kG}$ angegeben. Wir zeigen deshalb in Abb. 3 nur das Aufspaltungsschema der Linie C III $5696 \AA$.

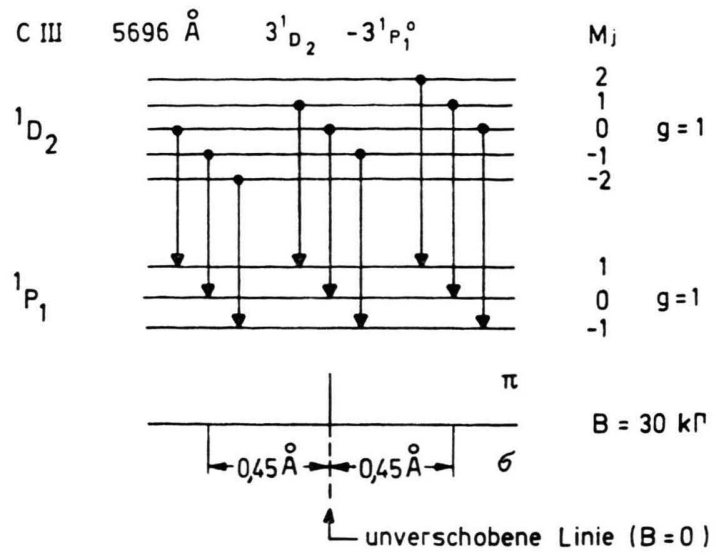

Abb. 3. Zeeman-Aufspaltung der Linie C III $5690 \AA$ bei einem Magnetfeld von $30 \mathrm{kG}$.

Für das Experiment wichtig ist auch die Polarisation der Zeeman-Komponenten, die sich aus quantenmechanischen Auswahlregeln bestimmt ${ }^{10}$ :

Beobachtungsrichtung $\perp$ zum Magnetfeldvektor $\boldsymbol{H}$ : linear polarisiert $\perp$ zu $\boldsymbol{H}$ : $\quad \sigma$-Komponenten; linear polarisiert || zu $\boldsymbol{H}: \quad \pi$-Komponenten; Beobachtungsrichtung || zum Magnetfeldvektor $\boldsymbol{H}$ : zirkular polarisiert $\sigma$-Komponenten.

An unserem Lichtbogen kommt aus technischen Gründen nur die Beobachtung side-on, d. h. senkrecht zum Vektor der magnetischen Feldstärke, in Frage. Mit Hilfe eines Polarisationsfilters lassen sich jeweils $\sigma$ - oder $\pi$-Komponenten herausfiltern.

\section{f) Doppler-Verbreiterung durch Rotation der Plasmasäule}

Die Messung der Doppler-Verbreiterung kann auch noch durch eine Rotation der Bogensäule stark gestört werden. MAHN, RINGLER und ZANKL ${ }^{3}$ haben eine solche Rotation beobachtet, die dadurch angefacht wurde, daß bei homogenem Magnetfeld der Strom in der Ringanode quer zu B fließen mußte, wodurch zur Rotation führende Lorentz-Kräfte entstanden.

9 L. D. LANDAu u. E. M. Lifschitz, Quantenmechanik, Akademie-Verlag, Berlin 1965.
Nach ${ }^{3}$ und ${ }^{11}$ ergaben sich Rotationsgeschwindigkeiten von etwa $3 \cdot 10^{6} \mathrm{~cm} / \mathrm{sec}$ in $1 \mathrm{~cm}$ Abstand von der Achse. Nimmt man an, daß die ganze Plasmasäule starr rotiert, so wächst die Doppler-Verschiebung vom Werte Null in der Achse nach außen proportional mit dem Radius $r$ an. Bei einem mittleren Radius von $8 \mathrm{~mm}$, bis zu dem die Emission von C III-Linien beobachtet wird, erhält man mit $\Delta \lambda / \lambda=v_{\varphi} / c$ und $\lambda=6000 \AA$ eine maximale Wellenlängenverschiebung von $\Delta \lambda=0,5 \AA$. Wegen der endlichen, experimentell möglichen, Ortsauflösung führt dies zu einer Linienverbreiterung, die in der Größenordnung des thermischen Doppler-Effekts liegt. Rotiert der Bogen nicht starr, so werden die Verhältnisse nicht grundsätzlich anders sein. Es sei hier schon darauf hingewiesen, daß die Rotationsgeschwindigkeit des von uns untersuchten Lichtbogens sicher um eine Größenordnung geringer war $(\mathrm{Ab}$ schnitt 3.2). Dies hängt mit der günstigen Stromführung innerhalb der Ringanode zusammen, wie sie oben beschrieben wurde.

\section{Messungen}

\subsection{Optische Anordnung und Meßverfahren}

Wir verwenden ein Fabry-Perot-Interferometer mit niedrigem Reflexionsvermögen der silberverspiegelten Platten $(r=0,7)$, aber hoher Transmission $(T=70 \%)$. Bei der Wellenlänge der C III-Linie $\lambda=5696 \AA$ beträgt der freie Spektralbereich $\Delta \lambda_{\mathrm{s}}=1,6 \AA$ (Plattenabstand $t=1 \mathrm{~mm}$ ) und das effektive Auflösungsvermögen $A_{\lambda}$ $=0,2 \AA$. Die Apparatefunktion bestimmten wir experimentell mit der Linie Cd $6438 \AA$ einer Hg-Cd-Niederdrucklampe und mit der Linie $6328 \AA$ eines kontinuierlichen He-Ne-Lasers.

Die optische Anordnung für die Messung der Doppler-Profile ist in Abb. 4 schematisch dargestellt. Die optische Abbildung wird so gewählt, daß eine Ortsauflösung möglich ist, d. h. jedem Interferenzring ein bestimmter Ort im Bogen zugeordnet werden kann. In vertikaler Richtung liefert das Interferenzmuster Information über die $z$-Achse der Plasmasäule. In horizontaler Richtung entspricht der innerste Ring mit dem Radius $R_{1}$ einem Ort $x_{1}$, der nächste Ring mit dem Radius $R_{2}$ einem weiter von der Achse entfernten Ort $x_{2}$ usw., entsprechend den Winkeln $\varphi_{1}, \varphi_{2}, \ldots$, unter denen das aus dem jeweiligen Bogenbereich stammende Lichtbündel auf die Platten des Etalons auftrifft.

Die Entladung wird „side-on“ auf zwei gekreuzte, in ihrer Breite veränderliche Spalte abgebildet, die ein

10 H. E. White, Introduction to Atomic Spectra, McGrawHill, New York 1934.

11 H.-F. Doebele, Z. Naturforsch. 24 a, 1249 [1969]. 


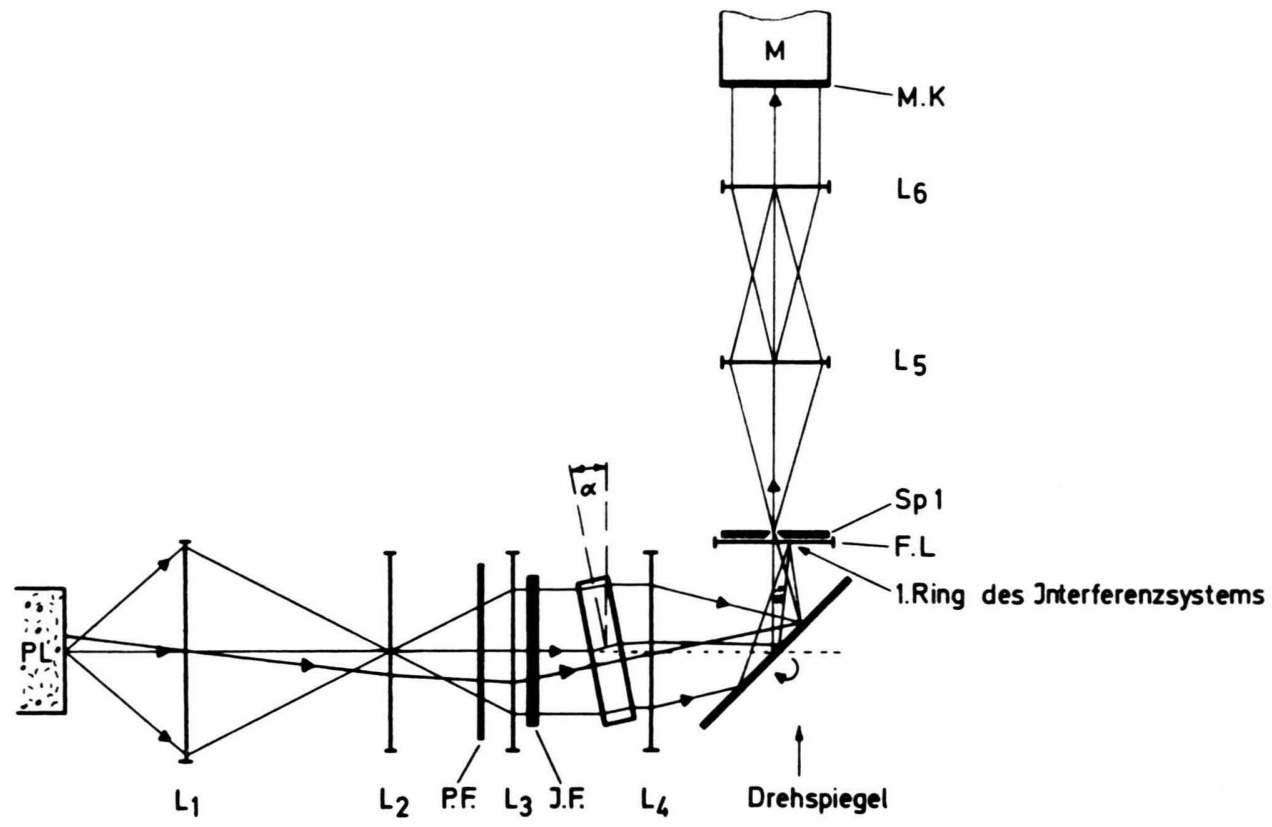

Abb. 4. Optische Anordnung (Abkürzungen: Pl Plasma; $\mathrm{L}_{1}, \mathrm{~L}_{2}, \ldots$ Linsen bzw. Objektive; P.F. Polarisationsfilter; I.F. Interferenzfilter; F.L. Feldlinse; Sp. 1 horizontaler Spalt [s. dazu Abb. 5]; M.K. Multiplier-Kathode; M Multiplier; $\alpha$ Winkel, um den das Fabry-Perot-Interferometer gegen das einfallende Lichtbündel geneigt ist).

kleines Segment aus dem Haidinger-Ringsystem ausschneiden. Ein vertikaler Spalt schneidet einen schmalen Streifen, dessen Breite etwa der Halbwertsbreite des Instrumentenprofils entspricht, aus dem Ring mit dem Radius $R_{\mathrm{i}}$ in $x$-Richtung aus (Abb. 5). Der hori-
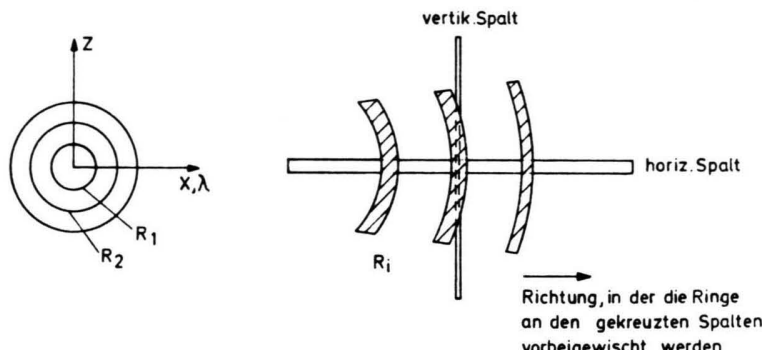

Abb. 5. Zur elektronischen Registrierung der Linienprofile.

zontale Spalt begrenzt den Ringausschnitt, dessen Profil gemessen werden soll, in $z$-Richtung und wird so schmal gehalten (etwa $1 \mathrm{~mm}$ breit), daß die Krümmung des Ringausschnitts vernachlässigbar ist. Mit dem Licht dieses Ringausschnitts wird durch die Linsen $\mathrm{L}_{5}$ und $\mathrm{L}_{6}$ die Kathode des Multipliers (EMI 9656 F) gleichmäBig ausgeleuchtet. Vor dem Fabry-Perot-Interferometer, das zwischen den Objektiven $\mathrm{L}_{3}$ und $\mathrm{L}_{4}$ im parallelen Licht steht (Abb. 4), befinden sich ein Polarisationsfilter zur Unterdrückung der $\sigma$-Zeeman-Komponenten und ein schmalbandiges Interferenzfilter, das nur eine Spektrallinie durchläßt. Die Filter für die Linien C IV
$5801 \AA$ und $5812 \AA$ haben eine maximale Durchlässigkeit von $40 \%$ bei einer Halbwertsbreite von $4 \AA$. Das Filter für die Linie C III $5696 \AA$ hat eine maximale Durchlässigkeit von $60 \%$ bei einer Halbwertsbreite von $10 \AA$.

Das ganze Ringsystem wird mit Hilfe eines Drehspiegels an den gekreuzten Spalten innerhalb der konstanten Phase einer Entladung „vorbeigewischt“ und elektronisch mit einem Oszillographen registriert. Wegen der geringen Intensität der Kohlenstofflinien können wir bei einer Entladung nur bis zu einem Radius von $4 \mathrm{~mm}$ die Profile elektronisch aufzeichnen. Für die Außenzonen des Bogens $(r>4 \mathrm{~mm})$ ersetzen wir das Interferenzfilter durch einen Spektrographen und machen Plattenaufnahmen mit $40-50$ Entladungen pro Aufnahme.

Um die Temperatur im achsennahen Bereich des Entladungskanals messen zu können, müssen wir das F.-P.

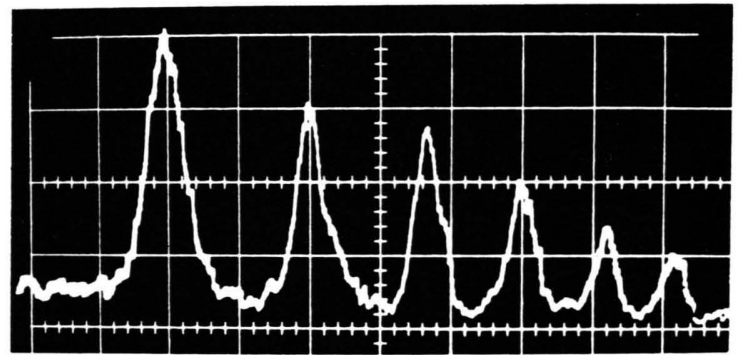

Abb. 6. Oszillogramm des Ringsystems der Linie C III $5696 \AA$. 
Interferometer so „kippen“, daß das Lichtbündel aus dem Gebiet um $r=0$ unter einem endlichen Winkel zur Normalen auf die Etalonplatten auftrifft. Durch die passende Wahl der Spiegel-Umdrehungsgeschwindigkeit erreichen wir, daß das Ringsystem in 0,5 sec elektronisch registriert wird (Dauer der stationären Phase der Entladung 0,8 sec).

Die Abb. 6 zeigt ein typisches Oszillogramm von den Profilmessungen der Linie C III $5696 \AA$ ( $\pi$-ZeemanKomponente).

\subsection{Untersuchung der Rotation des Bogens}

Eine eventuelle Rotation der Bogensäule wird durch Lorentz-Kräfte angetrieben, die aus der Wechselwirkung des Bogenstroms mit dem äußeren Magnetfeld resultieren. Polt man das Magnetfeld um, so muß auch der Bogen seinen Drehsinn ändern. Die Spektrallinie erscheint dann unsymmetrisch zu kurzen Wellenlängen hin verbreitert, da sich das Plasma in unserem Modell (Abb. 7) zum Beobachter hin bewegt. Wir können demnach den zweifachen Doppler-Effekt messen, wenn wir zwei Aufnahmen auf einen Film registrieren und das Magnetfeld nach der ersten Aufnahme umpolen. Wegen der geringen Intensität der Kohlenstofflinien verwenden wir zur Messung ein Fabry-Perot-Interfero-

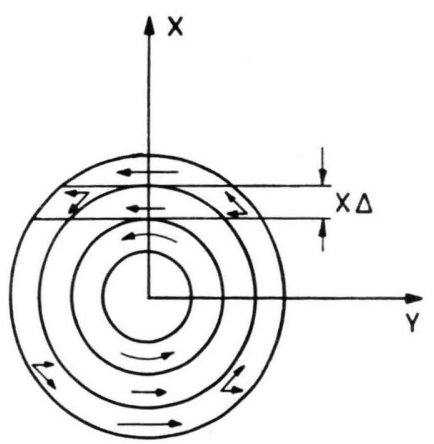

Beobachtungsrichtung

Abb. 7. Zur Linienverbreiterung durch Rotation. meter in Verbindung mit einem schmalbandigen Interferenzfilter für die betreffende Linie.

Wir interessieren uns besonders für die Rotationsgeschwindigkeiten in den inneren Zonen der Plasmasäule $(r<8 \mathrm{~mm})$. Da dort die thermische DopplerVerbreiterung jedoch einige Zehntel Ångström beträgt, können wir das Auflösungsvermögen des Interferometers nicht über $0,07 \AA$ hinaus steigern (Plattenabstand des Etalons 3,02 mm, nutzbarer Spektralbereich 0,53 $\AA$, C III $5696 \AA$ ), weil bei noch kleinerem Auflösevermögen der nutzbare Spektralbereich so klein wäre, daß sich die Interferenzordnungen überlappen. Eine Rotation des Bogens äußert sich beim F.-P.-Interferometer und der gewählten optischen Abbildung in einer Änderung der Radien der Interferenzkreise. Wir photographieren das Ringsystem der Linie C III $5696 \AA$ für $\pi$ und $\sigma$-Zeeman-Komponenten in jeder Magnetfeldrichtung getrennt. Dann belichten wir jeweils zwei (oder Vielfache von 2) Aufnahmen auf ein Bild und polen zwischen den Aufnahmen das Magnetfeld um.

Abb. 8 zeigt zwei Photographien des Ringsystems der $\pi$-Zeeman-Komponente der Linie C III $5696 \AA$.

Die photometrische Bestimmung der Radien der Interferenzringe bei den Einzelaufnahmen und die Registrierung der Profile bei den übereinanderbelichteten Aufnahmen ergibt übereinstimmend, daß innerhalb der Meßgenauigkeit von etwa 5\% keine Verschiebung der Interferenzkreise beim Umpolen des Magnetfeldes auftritt.

Wir schließen daraus, daß Doppler-Verschiebungen infolge Rotation des Bogens größer als 0,03 $\AA$ nicht vorkommen, d.h., daß die Rotationsgeschwindigkeit höchstens $1,6 \cdot 10^{5} \mathrm{~cm} / \mathrm{sec}$ bei einem mittleren Radius von $6 \mathrm{~mm}$ beträgt.

\subsection{Auswertung der Meßdaten}

Die Linienprofile sind auf Grund der linearen Dispersion $D_{1}$ des F.-P.-Interferometers verzerrt. Mit $\left|D_{1}\right|=|\mathrm{d} R| /|\mathrm{d} \lambda|=f^{2} / R \cdot \lambda_{0} \quad(f=$ Brennweite des abbildenden Objektivs; $R=$ Radius des Interferenzrin-
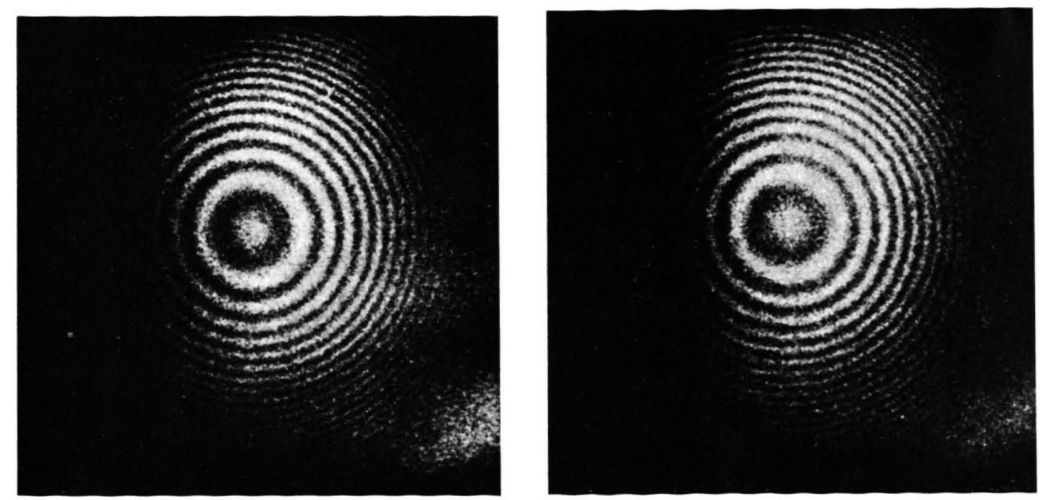

Abb. 8. Ringsystem der $\pi$-Zeeman-Komponente der Linie C III 5696 A. Linkes Bild: $\boldsymbol{B}$ zwischen zwei Aufnahmen umgepolt. Rechtes Bild: $\boldsymbol{B}$ nicht umgepolt. 
ges; $\lambda_{0}=$ Wellenläge der verwendeten Spektrallinie) folgt daraus:

$$
\Delta \lambda=\int_{R_{0}}^{R_{0}+\Delta R} \frac{1}{D_{1}} \mathrm{~d} R=\frac{\lambda_{0}}{2 f^{2}}\left(2 R_{0} \Delta R+(\Delta R)^{2}\right)
$$

( $R_{0}$ ist der Anfangspunkt des Entzerrungsvorganges).

Die Radien der Interferenzkreise werden photometrisch aus Photographien des Ringsystems bestimmt. Die Profile auf den Oszillogrammen bzw. Plattenaufnahmen werden mit Hilfe der Gl. (4) auf Wellenlängeneinheiten umgerechnet. Die so gewonnenen Profile entstehen durch Faltung der wahren Linienprofile mit der Apparatefunktion des Interferometers. Die Entfaltung beschreiben wir im Anhang. Außerdem müssen die bei der side-on-Beobachtung im Abstand $x$ von der Bogenachse erhaltenen Strahlungsintensitäten noch mit Hilfe der Abelschen Integralgleichung auf radiale Werte umgerechnet werden. Diese Inversion wird numerisch mit einem vorhandenen Rechenprogramm durchgeführt ${ }^{2}$.

\section{Ergebnisse und Diskussion}

Wenn die gemessenen Linienprofile $L(r, \lambda)$ durch den thermischen Doppler-Effekt bestimmt werden, müssen sie die Form einer Gauß-Glocke haben. Um diese Eigenschaft nachzuprüfen, werden Profile für verschiedene Orte $r$ als Funktion von $(\Delta \lambda)^{2}$ in logarithmischem Maßstab aufgetragen $(\Delta \lambda=$ Abstand von der Linienmitte). Da dieser Vorgang in guter Näherung zu Geraden führt, können wir schließen, daß unsere endgültigen Profile gaußartig sind. Aus den Halbwertsbreiten der Doppler-verbreiternden

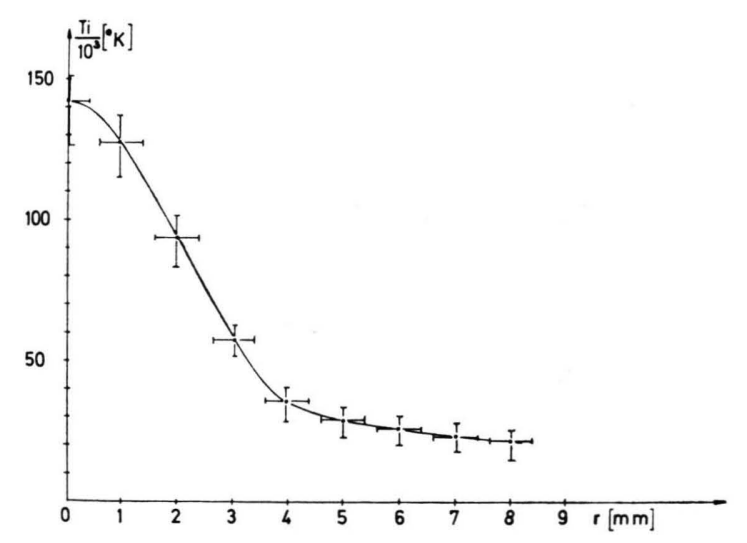

Ab. 9. Radiales Profil der Ionentemperatur.
Profile können wir nach Gl. (3) die radiale Ionentemperaturverteilung ausrechnen. Das gemittelte Ergebnis aus 5 Einzelmessungen ist in Abb. 9 aufgetragen. Die angegebenen Werte für den Bereich von $r=0$ bis $r=4 \mathrm{~mm}$ stammen aus elektronischen Messungen, die Werte für $r>4 \mathrm{~mm}$ aus Plattenaufnahmen.

\subsection{Fehlerabschätzung}

Die bei Tichmann ${ }^{2}$ in das Rechenprogramm zur Berechnung der Abelschen Integralgleichung eingebaute Fehlerrechnung basiert auf der Annahme, daß der statistische Fehler der Gesamtintensität für bestimmte Abstände $x$ von der Bogenachse angegeben werden kann. Da wir Linienprofile in Abhängigkeit von $x$ gemessen haben, liegen die Verhältnisse sehr viel komplizierter. Die Linienprofile mußten entzerrt und entfaltet werden, bevor sie „entabelt“ werden konnten. Eine mathematisch exakte Fehlerrechnung ließe sich daher nur mit großem Aufwand durchführen. Wir beschränken uns deshalb darauf, Größtfehler anzugeben. Wir entnehmen den elektronischen Aufnahmen Mittelwerte, Minimal- und Maximalwerte. Beim Entzerrungsvorgang berücksichtigen wir einen Fehler, der durch die Festlegung des Anfangspunktes $R_{\mathbf{0}}$ und die Umrechnung der Strahllaufzeit in Dispersion des Ringsystems entstehen kann. Bei der Entfaltung der Profile liegt die größte Unsicherheit in der Anpassung der Linienflügel. Der Einfluß der Flügel auf die für uns entscheidende Halbwertsbreite der Linien ist jedoch gering. Für die nun vorliegenden Mittelwerte, oberen und unteren Grenzwerte der entfalteten Profile führen wir die AbelInversion getrennt durch. Der Abstand der Kurven, die dem oberen bzw. unteren Grenzwert entsprechen, gibt den Größtfehler an. Die mögliche, mit unseren Mitteln aber nicht erkennbare Rotationsgeschwindigkeit von $1,6 \cdot 10^{5} \mathrm{~cm} / \mathrm{sec}$ rechnen wir als scheinbare Temperaturerhöhung (infolge einer scheinbaren Verbreiterung der gesamten Spektrallinie um maximal $0,03 \AA$ ) von $2-10 \%$ - je nach dem zugrunde liegenden Profil - bei der Größtfehlerangabe ein.

Es ergeben sich insgesamt Größtfehler für die Halbwertsbreiten der Linien zwischen $10 \%$ und $15 \%$. Daraus folgen für die Ionentemperatur (wegen $T \sim \sqrt{\text { Halbwertsbreite }) ~ F e h l e r ~ z w i s c h e n ~ 20 \% ~ u n d ~}$ $30 \%$. Die Zuordnung der Temperatur zu einem bestimmten Bogenradius $r$ ist nur mit einer Unsicherheit von $\pm 0,4 \mathrm{~mm}$ möglich. 


\subsection{Diskussion des Temperaturprofils}

Das gemessene radiale Profil der Ionentemperatur zeigt nicht den bei Wasserstoffplasma üblichen Übergang von Zonen mit steilen Temperaturgradienten zu einer flachen Schulter, die im Bereich der teilweisen Ionisation im wesentlichen durch die Reaktionswärmeleitfähigkeit ${ }^{12}$ verursacht wird.

Örtliche und zeitliche Schwankungen im Plasma während der etwa 50 Entladungen, die für eine Aufnahme notwendig waren, können wahrscheinlich für die Abweichung von dem gewohnten Verlauf des Temperaturprofils in den Außenbereichen des Bogens verantwortlich gemacht werden.

Wenn wir die bei unseren Parametern (elektrische Feldstärke einige Volt/cm, Druck etwa 190 Torr) vernünftige Annahme machen, da $T_{\mathrm{e}} \approx T_{\mathrm{i}}$ ist, so können wir unsere Messungen mit denen von ${ }^{3}$ vergleichen. In unserem Fall waren das Magnetfeld etwa um den Faktor 3, der Bogenstrom um den Faktor 1,5 und die Bogenlänge um den Faktor 5 größer als bei ${ }^{3}$. Die Erhöhung des Stromes führt zu einer stärkeren Heizung des Plasmas, während die Vergrößerung von Magnetfeld und Bogenlänge die Verluste vermindert. Insgesamt gesehen, erscheint daher die bei uns gemessene Achsentemperatur von $1,4 \cdot 10^{5}{ }^{\circ} \mathrm{K}$ gegenüber der Temperatur von $1 \cdot 10^{5}{ }^{\circ} \mathrm{K}$ bei ${ }^{3}$ plausibel.

Die Begründung für den unerwartet glatten Verlauf der Abel-Inversion für die achsennahen Zonen liegt wohl im Verlauf des Emissionskoeffizienten, der für die C III-Linie bei $\lambda=5696 \AA$ sein Maximum auf der Bogenachse erreicht. Zwar ist bei unserer Achsentemperatur das Strahlungsmaximum für diese Linie bereits überschritten, doch wahrscheinlich sind Relaxationserscheinungen (siehe dazu ${ }^{1}$ und ${ }^{2}$ ) für diese räumliche Verschleppung verantwortlich.

\section{Zusammenfassung und Schluß}

Die Bestimmung der Ionentemperatur aus der Halbwertsbreite Doppler-verbreiterter Spektrallinien ist (bei side-on-Beobachtung) in vernünftigen Fehlergrenzen an einer Plasmasäule möglich, deren Rotationsgeschwindigkeit so klein ist, $\mathrm{da} ß$ die daraus folgende Doppler-Verbreiterung gegen den thermischen Doppler-Effekt vernachlässigbar ist. Wenn die

12 R. W IENECKE, Z. Naturforsch. 19 a, 676 [1964].

13 L. Fox u. E. T. Goodwin, Phil. Trans. A 245, 501 [1953].
Rotationsgeschwindigkeit groß ist, muß die radiale Geschwindigkeitsverteilung vorliegen, damit man den makroskopischen vom thermischen DopplerEffekt eindeutig trennen kann. Das Ergebnis der Abel-Inversion für den achsennahen Bereich hängt bei side-on-Beobachtung entscheidend vom Verlauf des radialen Emissionskoeffizienten der Spektrallinie ab, die zur Messung verwendet wird. Die Fehler bei der Inversion werden klein, wenn dieser Emissionskoeffizient sein Maximum auf der Bogenachse annimmt.

Für die Anregung zu dieser Arbeit sowie für dauerndes Interesse bei ihrer Durchführung möchten wir Herrn Prof. Dr. R. WIENECKE sehr danken.

\section{Anhang \\ Entfaltung der gemessenen Profile}

Eine monochromatische Linie wird durch die Apparatefunktion $k(x)$ zu einer Intensitätsverteilung verschmiert. Das scheinbare Profil $g(x)$, das wir messen, entsteht durch Faltung des wahren Profils der Linie $f(x)$ mit dem Apparateprofil $k(x)$. Mathematisch läßt sich dieser Sachverhalt durch folgende Integralgleichung ausdrücken ( $x$ und $y$ in Wellenlängen- oder Frequenzeinheiten) :

$$
g(x)=\int_{-\infty}^{+\infty} f(y) k(x-y) \mathrm{d} y .
$$

Gl. (5) ist eine singuläre Fredholmsche Integralgleichung erster Art. Sie heißt singulär, weil die Integrationsgrenzen unendlich sind. Gl. (5) ist vom Faltungstyp, da der Kern nur von der Differenz $(x-y)$ der Quell- und Aufpunktskoordinaten abhängt.

Bei der numerischen Integration müssen die Integrationsgrenzen als endlich angenommen werden:

$$
\int_{-1}^{+1} k(x-y) f(y) \mathrm{d} y=g(x)
$$

(die Normierung ist so gewählt, daß die Integration von -1 bis +1 läuft).

Im allgemeinen existieren keine eindeutigen Lösungen dieser nunmehr nicht-singulären Fredholmschen Integralgleichung erster Art (siehe z. B. ${ }^{13}$ und ${ }^{14}$ ). Verwendet man eine numerische Integrationsformel zur Lösung der Gl. (6), so treten bei der Lösung oft Oszillationen mit großer Amplitude und kurzer Periode auf, wenn die Schrittweiten klein sind. Fox und GoodwIN haben diese Frage geprüft und haben gefunden, daß $f(y)$ oszillieren kann, ohne daß dadurch in $g(x)$ wesentliche Änderungen hervorgerufen werden. Aus diesem Verhalten der Integralgleichung folgt dann, daß wir bei einer etwa auftretenden glatten Lösung nicht in der

14 F. G. Tricomi, Integral Equations, Intersience Publishers, New York-London 1957. 
Lage sind, die Abweichungen von der wahren Funktion $f(y)$ anzugeben. Wir beschreiten daher einen anderen Weg, um das wahre Profil $f(x)$ aus dem gemessenen, scheinbaren Profil $g(x)$ zu erhalten:

Die Linien, die wir messen, sind durch den thermischen Doppler-Effekt verbreitert. Sie besitzen daher an ihrem Entstehungsort Gauß-Profile. Wir führen jedoch unsere Messungen am Lichtbogen side-on aus, d. h. wir messen an einem Ort $x$ ein Linienprofil, das aus einer Überlagerung verschiedener Gauß-Profile in Abhängigkeit von $r$ entsteht.

Wir nehmen im ersten Schritt an, daß das scheinbare Profil $g(x)$ das Ergebnis der Faltung eines Gauß-Profils $f^{*}(y)$ mit der gemessenen Apparatefunktion $k(x)$ ist.

Wir konnten die gemessene Apparatefunktion hinreichend gut durch ein theoretisches Airy-Profil annähern. Daraus folgt dann der reguläre symmetrische Integralkern :

$$
k(x-y)=\frac{1}{1+R \sin ^{2}\left[\frac{1}{2} \pi(x-y)\right]} .
$$

$\left(R=4 r_{\text {eff }} /\left(1-r_{\text {eff }}\right)^{2} ; r_{\text {eff }}=\right.$ effektives Reflexionsvermögen des Fabry-Perot).

Eine solche Funktion $f^{*}(y)$ wählen wir auf folgende Weise: MINKOwSKI und BRUCK ${ }^{15}$ haben das Ergebnis der Faltung von Doppler- und Airy-Profilen in Abhängigkeit des Verhältnisses ihrer Halbwertsbreiten graphisch dargestellt. Wenn man das Verhältnis der Halbwertsbreiten der gemessenen Funktionen $g(x)$ und $k(x-y)$ kennt, kann man dort die Halbwertsbreite der zugrunde liegenden Funktion $f^{*}(y)$ ablesen. Mit dieser Funktion $f^{*}(y)$ führen wir das Integral in Gl. (6) numerisch aus. Die Übereinstimmung der so berechneten $g^{*}(x)$ mit den gemessenen $g(x)$ ist nur in wenigen Fällen befriedigend. Eine deutliche Verbesserung der Approximation erreichen wir, indem wir $f^{*}(y)$ durch eine Summe von Gauß-Profilen ersetzen

$$
f^{*}(y) \rightarrow f^{* *}(x)=\sum_{i=1}^{n} a_{i} e^{-x^{2} / b_{i}{ }^{2}} .
$$

Bei den meisten mit dieser Methode angenäherten scheinbaren Profilen genügten wenige Glieder der Summe (8), um eine Übereinstimmung von besser als $3 \%$ zu erhalten.

Die Abb. 10 zeigt drei Profile, die repräsentativ für die gesamte Meßreihe sind.

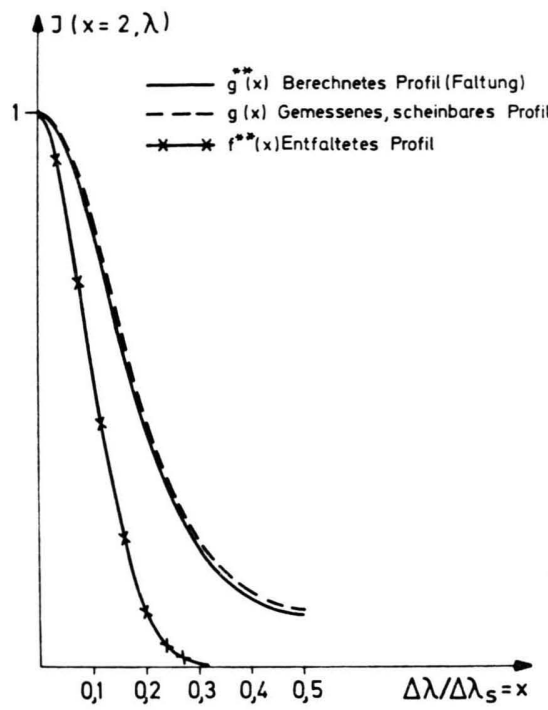

Abb. 10. Zur Entfaltung der gemessenen Linienprofile.

Diese Arbeit wurde im Rahmen des Vertrages zwischen dem Institut für Plasmaphysik und der Europäischen Atomgemeinschaft über die Zusammenarbeit auf dem Gebiete der Plasmaphysik durchgeführt.

15 R. Minkowsky u. H. Bruck, Z. Phys. 95, 274 [1935]. 